\title{
Effects of Imidazole- and Triazole-derivative Antifungal Compounds on the Growth and Morphological Development of Candida albicans Hyphae
}

\author{
By F. C. ODDS, ${ }^{*}$ A. COCKAYNE, $\dagger$ J. HAYWARD AND A. B. ABBOTT \\ Department of Microbiology, University of Leicester, Leicester LE1 7RH, UK
}

(Received 28 February 1985; revised 21 May 1985)

\begin{abstract}
Six azole-derivative antifungal compounds affected several aspects of Candida albicans hyphal development with only a relatively small degree of inhibition of growth rate, measured in terms of ATP concentration, whereas amphotericin B and 5-fluorocytosine affected morphology only when they also substantially inhibited fungal growth rate. At $10^{-8} \mathrm{M}$, all the azoles tested inhibited branch formation by $C$. albicans hyphae. At $10^{-7} \mathrm{M}$ and higher concentrations, clotrimazole and miconazole strongly suppressed emergence of new hyphal outgrowths from parent yeast cells, whereas ICI 153066 and itraconazole had little effect on this phenomenon and ketoconazole and tioconazole had intermediate effects. At the highest concentrations tested $\left(10^{-5} \mathrm{M}\right)$ hyphal development was ultimately arrested by the azole compounds and the fungus grew predominantly in the form of budding yeast cells; however, none of the azole antifungals prevented initial emergence of an apparently normal germ tube. The antifungals only exerted their morphological effects when they were present in the culture medium: removal of the compounds after exposure of $C$. albicans to them led to reversion to normal growth.
\end{abstract}

\section{INTRODUCTION}

Various imidazole-derivative antifungal agents have been claimed to inhibit the morphological transformation of Candida albicans from budding yeast cells to hyphae (Borgers et al., 1979; Borgers, 1980; Davies \& Marriott, 1981). However, this inhibitory effect is not unequivocal. Johnson et al. (1982) were unable to detect any inhibition of germ tube outgrowth by ketoconazole in serum, and Davies \& Marriott (1981) saw inhibition of hyphal extension by low concentrations of tioconazole or ketoconazole only when the drugs were added with the yeast inoculum: there was no inhibition of extension of preformed germ tubes even when drugs were added at 100 times the concentration that inhibited germ tube initiation.

Since substances with definable and specific effects on morphological changes in $C$. albicans would be of obvious value in elucidating the mechanisms determining such changes, there is a case for further study of the effects of imidazole antifungals and the newer triazole compounds on C. albicans morphogenesis. Most investigators of morphological change in this fungus have studied only the early stages of the process - emergence of hyphal germ tubes from stationary phase yeast cells - although later events in hyphal development, such as formation of hyphal branches or lateral buds from sites adjacent to hyphal septa, also merit attention as morphogenetic processes (Odds, 1985). The present study was therefore undertaken to establish whether one or more of several antifungal azole compounds might be sufficiently selective in its effects on morphogenetic processes to be a useful probe for further study of the processes. The study was confined to relatively low concentrations of antifungals since the aim was to demonstrate possible morphological effects that were detectable in cells whose overall growth rate was unaltered by the antifungals.

† Present address: Department of Microbiology, University of Birmingham, Birmingham B15 2TT, UK.

Abbretiation: EMEM, Eagle's minimal essential medium. 


\section{METHODS}

Fungi and growth conditions. C. albicans isolate 73/055 (originally obtained from a vaginal swab) was used in the experiments. The fungus was maintained on Sabouraud's dextrose agar (Oxoid) and inocula were grown overnight in this medium and prepared by suspension of the yeast cells in sterile water. Concentrations of yeast cells were determined by haemocytometer counts.

C. albicans was grown in its hyphal form in Eagle's minimal essential medium (EMEM) containing glutamine (Gibco) buffered at pH 7.3 with $0.02 \mathrm{M}$-HEPES and $\mathrm{NaHCO}_{3}$ and incubated at $37^{\circ} \mathrm{C}$ under $5 \% \mathrm{CO}_{2}$ in air. Serum was not included in the medium since the object of the experiments was to allow inhibitory compounds to express their effects on morphology maximally; protein binding of the compounds might be expected to complicate the experiments. The medium was prewarmed to $37^{\circ} \mathrm{C}$, inoculated to a yeast cell concentration of $10^{6} \mathrm{ml}^{-1}$, dispensed in $1 \mathrm{ml}$ lots in the wells of Repli-dishes (Sterilin) and incubated statically. Unless otherwise stated, antifungal stock solutions or their solvents were added to the medium just before inoculation. Samples of cultures were taken at intervals by resuspension of the contents of a Repli-dish well with a magnetic stirrer and a small wire follower (Odds, 1982). Samples $(0.5 \mathrm{ml})$ were treated immediately for extraction of ATP (see below). To the remaining $0.5 \mathrm{ml}$ was added $50 \mu \mathrm{l}$ of preservative [sodium dodecyl sulphate, $100 \mathrm{~g} \mathrm{l}^{-1}$ in $10 \%(\mathrm{v} / \mathrm{v})$ formalin] and the samples were stored at room temperature for subsequent microscopic examination.

In experiments where antifungal drugs were removed from cultures at intervals after inoculation, the procedure was as follows. Cultures $(10 \mathrm{ml})$ were set up in $25 \mathrm{ml}$ glass universal bottles. At the time of drug removal the cultures were centrifuged at $2500 \mathrm{~g}$ for $3 \mathrm{~min}$, the supernatants were discarded and the fungi were washed in antifungal-free EMEM then resuspended in $10 \mathrm{ml}$ antifungal-free EMEM. Parallel cultures were washed and resuspended in fresh medium containing the same antifungal concentration as before to act as controls for the centrifugation/resuspension procedures. Results from the latter showed there was no measurable effect of the procedures on the growth rates or morphology of $C$. albicans.

Antifungal compounds. Six azole antifungals and two non-azole compounds were tested for their effects on $C$. albicans growth and morphology. Clotrimazole (Bayer), ICI 153066 (ICI Pharmaceuticals), itraconazole, ketoconazole and miconazole (Janssen Pharmaceutical), and tioconazole (Pfizer) were kindly supplied by their manufacturers. Amphotericin B and 5-fluorocytosine were purchased from Sigma. The antifungals were prepared as stock solutions at 100 times their final test concentrations in DMSO and stored at $-20^{\circ} \mathrm{C}$ when not in use.

Measurement of biomass and morphology of C. albicans. Preliminary experiments showed that the effects of azole antifungals were reflected predominantly in altered dimensions of the $C$. albicans hyphae, numbers of branches per mycelium, numbers of yeast cells per mycelium, numbers of hyphal outgrowths per inoculum yeast cell, and hyphal growth rate (in terms of increase in ATP content). These parameters were therefore assessed for all samples.

Growth rates were determined by a least-squares regression analysis of plots of log fungal ATP concentration versus time. ATP was extracted from the fungal cells and ATP hydrolases inactivated by addition of $0.5 \mathrm{ml}$ samples to $5 \mathrm{ml}$ lots of Tris/EDTA buffer heated in a boiling water bath (Odds, 1982). ATP concentrations were determined by bioluminescence photometry (Odds, 1982). ATP concentrations in C. albicans have been shown to correlate well with alternative estimations of viable biomass (Ansehn \& Nilsson, 1984; F. C. Odds, unpublished data).

Hyphal lengths and widths were determined by phase-contrast microscopic examination of 25 mycelia from each sample with the aid of a calibrated eyepiece graticule. Only the lengths of primary hyphae were measured, i.e. those that appeared to be longest and attached to a large, phase-bright yeast cell, presumed to be the original yeast parent cell from the inoculum. In some drug-treated cultures differentiation between primary hyphal elements and other, pleomorphic types of growth was inevitably subjective (see e.g. Figs $1 b$ and $1 c$ ).

Numbers of hyphal branches were estimated for the same 25 mycelia that were used for determination of hyphal length, and the number of branches per hypha was calculated. Numbers of hyphal outgrowths per parent yeast cell were determined separately by counting a minimum of 30 mycelia, and the number of yeast cells (other than parent yeast cells) per mycelium was assessed on the basis of haemocytometer counts. Reductions in growth rate, mean hyphal length, hyphal outgrowths per parent yeast cell and branches per mycelium were calculated for each culture as percentages relative to the data for drug-free controls.

\section{RESULTS}

\section{Microscopic appearance of drug-treated cultures}

In antifungal-free cultures in EMEM the pattern of growth of $C$. albicans was typical of the hyphal growth form of the fungus. After $1 \mathrm{~h}$ germ tubes had emerged from most of the inoculum yeast cells. The germ tubes elongated into hyphae, and the earliest hyphal branches were formed after 3-4 h. The hyphae were predominantly phase-dark, but the parent yeast cells remained phase-bright throughout the observation period. By $5 \mathrm{~h}$ secondary hyphal shoots had formed from approximately $80 \%$ of the parent yeast cells. 

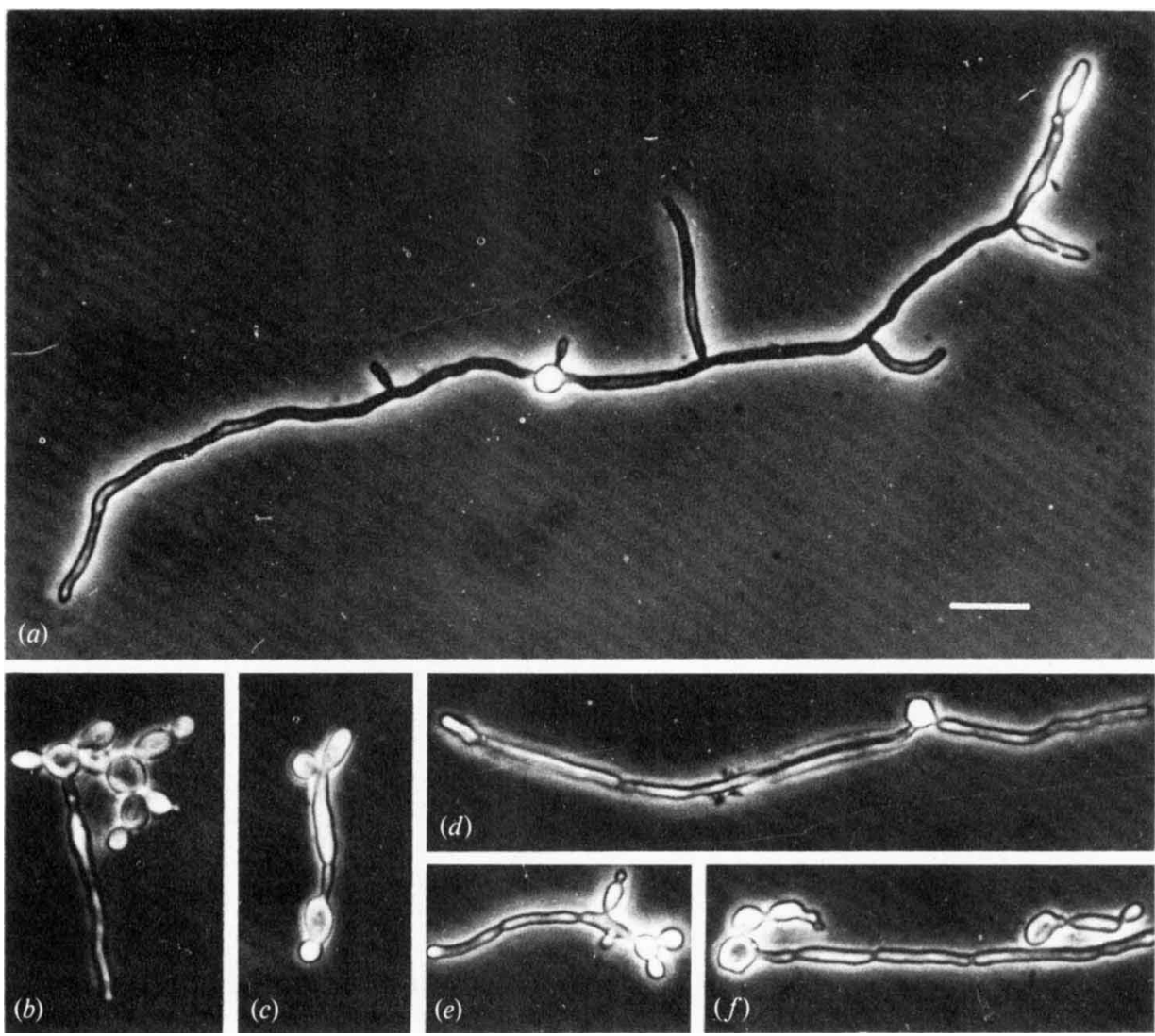

Fig. 1. Phase-contrast micrographs of $C$. albicans grown in EMEM under $5 \% \mathrm{CO}_{2}$ in air in the presence and absence of azole antifungals as follows. (a) No antifungal added; hyphae are phase-dark, branched and more than one hyphal outgrowth has emerged from the parent yeast cell. Bar, $10 \mu \mathrm{m}$. $(b)$ With $10^{-5} \mathrm{M}$-ketoconazole, $(c)$ with $10^{-6} \mathrm{M}$-clotrimazole, $(d)$ with $10^{-7} \mathrm{M}$-itraconazole, $(e)$ with $10^{-7} \mathrm{M}^{-}$ tioconazole and $(f)$ with $10^{-7} \mathrm{M}-$ miconazole.

The gross effects of azole antifungals on $C$. albicans hyphal morphology could be seen by qualitative microscopic observation of the cultures. For all the azole compounds tested there was a tendency for cells grown in the presence even of drug concentrations as low as $10^{-8} \mathrm{M}$ to appear phase-bright, unlike the control hyphae (Figs $1 a$ to $1 f$ ). A second effect common to the azole antifungals was a substantial inhibition of hyphal branching: hyphae grown in the presence of azoles consistently emerged as phase-dark germ tubes in the same manner as control hyphae during the first $2-3 \mathrm{~h}$ of growth, but in their subsequent development they turned pleomorphic and generated lateral buds instead of hyphal branches (Figs $1 c$ and $1 e$ ). At the highest azole concentrations hyphal development was sometimes arrested substantially after initial germ tube formation, and growth continued in the form of budding yeast cells that commonly remained in clumps.

Another effect of the azole antifungals was seen with some but not all of the compounds, and was most evident at the highest drug concentrations tested. This effect was an apparent failure of the original parent yeast cells to initiate new hyphal outgrowths - predominantly budding cells were formed from the parent after the initial (first-generation) germ tube emerged (Fig. $1 b$ ). In general, at the two highest drug concentrations tested $\left(10^{-5}\right.$ and $\left.10^{-6} \mathrm{M}\right)$ hyphal development appeared to be virtually abolished after an initial germ tube outgrowth, whereas at $10^{-7}$ and $10^{-8} \mathrm{M}$ hyphal extension appeared to be retarded, and the hyphal shape was less regular than in controls, but hyphal development was not arrested completely. 


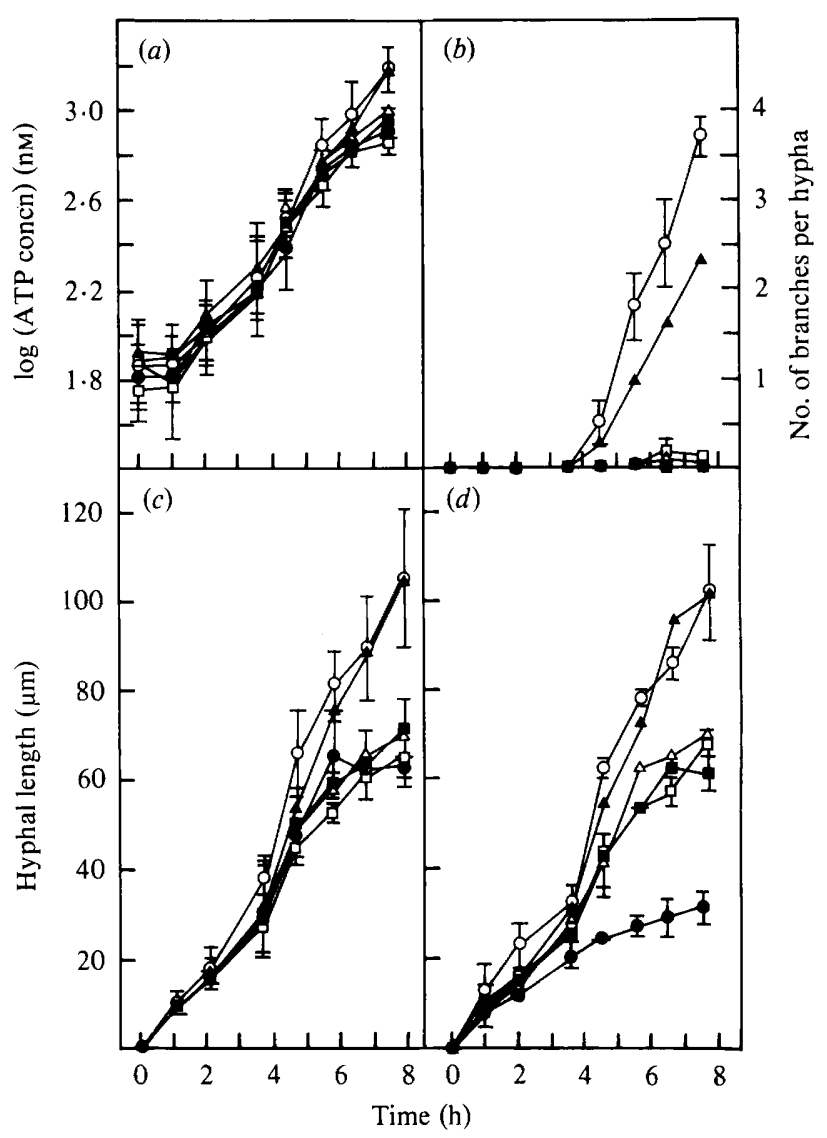

Fig. 2. Effects of azole antifungals on morphological development of C. albicans 73/055. (a) Effects of itraconazole on the hyphal growth curve, measured in terms of cellular ATP concentration; the effects of the other azole compounds tested were essentially the same. (b) Effects of tioconazole on hyphal branching; similar curves were obtained for all the azole antifungals tested. $(c, d)$ Effects of itraconazole and tioconazole respectively on the lengths of hyphae. The effects were similar over the concentration range $10^{-9}$ to $10^{-6} \mathrm{M}$, but tioconazole had a greater inhibitory effect on hyphal length at $10^{-5} \mathrm{M}$. whereas the effects of itraconazole appeared to be similar at this concentration. Cultures were treated with various concentrations of antifungals as follows: $\bigcirc$, control (no antifungal);, $10^{-5} \mathrm{M} ; \square, 10^{-6} \mathrm{M} ; \boldsymbol{\square}$, $10^{-7} \mathrm{M}: \triangle, 10^{-8} \mathrm{M}: \mathbf{\Delta}, 10^{-9} \mathrm{M}$. Bars indicate standard deviations for experiments done with three or more replicates.

\section{Effects of antifungals on rates of hyphal ATP increase}

Azole antifungals at concentrations from $10^{-9}$ to $10^{-5} \mathrm{M}$ appeared to have little or no effect on the growth curves of $C$. albicans hyphae, where ATP concentration was used as a measure of fungal biomass (Fig. $2 a$ ), although regression analysis of the growth curves revealed there was in fact some inhibition of growth rate at all test concentrations above $10^{-9} \mathrm{M}$ (Table 1). By contrast, amphotericin $\mathrm{B}$ was entirely non-inhibitory at $10^{-7} \mathrm{M}$ and entirely inhibitory at higher concentrations (Table 1). Only clotrimazole and ketoconazole appeared to cause any inhibition of biomass growth rates at $10^{-9} \mathrm{M}$, and, except for ICI 153066 , the extent to which growth rates were reduced by azole concentrations from $10^{-8}$ to $10^{-6} \mathrm{M}$ was essentially similar and virtually independent of dose-related effects. A generally greater degree of growth rate inhibition was evident at drug concentrations of $10^{-5} \mathrm{M}$. ICI 153066 was rather more inhibitory than the other azole compounds at concentrations from $10^{-8}$ to $10^{-5} \mathrm{M}$. 5-Fluorocytosine inhibited growth rate to an extent that was proportional to the drug concentration from $10^{-7}$ to $10^{-5} \mathrm{M}$. 
Table 1. Effects of antifungal drugs on rate of increase of ATP concentration (biomass growth rate) in C. albicans hyphae

The values are mean percentage decreases from the rate of ATP increase for drug-free control cultures, calculated by regression analysis of growth curve data for two or more replicate experiments. At least three replicate experiments were done with drug concentrations from $10^{-7} \mathrm{M}$ to $10^{-5} \mathrm{M}$. Standard deviations ranged from $4^{\circ}$ to $14^{\circ}$, iNT, not tested.

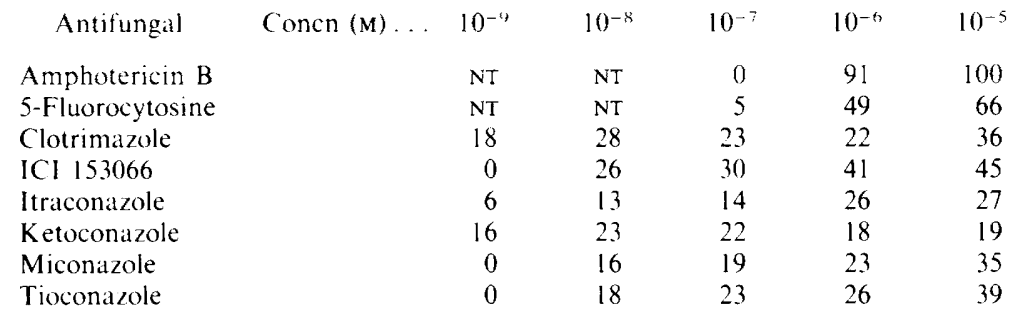

\section{Table 2. Effects of antifungal drugs on length of C. albicans hyphae after $7.5 \mathrm{~h}$ growth in EMEM}

The values are percentage reductions relative to drug-free control hyphae. For details of replication data see Table 1. Standard deviations ranged from 0 to $9 \%$ : NT, not tested.

\begin{tabular}{|c|c|c|c|c|c|c|}
\hline Antifungal & Conen $(M)$. & $10^{-49}$ & $10^{-8}$ & $10^{-7}$ & $10^{-6}$ & $10^{-5}$ \\
\hline Amphotericin B & & NT & NT & 0 & 88 & 100 \\
\hline 5-Fluorocytosine & & NT & $\mathrm{NT}$ & 0 & 37 & 66 \\
\hline Clotrimazole & & 0 & 33 & 40 & 34 & 57 \\
\hline ICI 153066 & & 0 & 27 & 39 & 41 & 47 \\
\hline Itraconazole & & 0 & 19 & 28 & 36 & 37 \\
\hline Ketoconazole & & 24 & 29 & 32 & 30 & 47 \\
\hline Miconazole & & 0 & 35 & 38 & 45 & 67 \\
\hline Tioconazole & & 11 & 39 & 35 & 39 & 67 \\
\hline
\end{tabular}

\section{Effects of antifungals on hyphal length}

The inhibitory effects of the antifungals on hyphal length correlated broadly with their effects on rates of ATP increase (Table 2). Detailed results are shown for two of the test antifungals in Figs $2(c)$ and $2(d)$. Itraconazole and tioconazole caused no reduction in hyphal length at $10^{-9} \mathrm{M}$, then essentially similar reductions in hyphal length from $10^{-8}$ to $10^{-6} \mathrm{M}$ (Figs $2 c$ and $2 d$ ). At $10^{-5} \mathrm{M}$, tioconazole caused a greater reduction in hyphal length than at $10^{-6} \mathrm{M}$ and lower concentrations, while the inhibitory effects of $10^{-5} \mathrm{M}$-itraconazole were little different from those at the lower concentrations. The other four azole antifungals inhibited hyphal lengths in patterns similar to those of either tioconazole or itraconazole (Table 2). Thus there were negligible effects at $10^{-9} \mathrm{M}$, a roughly equal inhibitory effect from $10^{-8}$ to $10^{-6} \mathrm{M}$, and differences only at $10^{-5} \mathrm{M}$, where clotrimazole, ketoconazole, miconazole and tioconazole reduced hyphal length by at least $50 \%$ more than at lower concentrations, whereas ICI 153066 and itraconazole were only 3 and $15 \%$, respectively, more inhibitory at $10^{-5} \mathrm{M}$ than at lower concentrations (Table 2).

The data concerning percentage inhibition of hyphal length for amphotericin B and 5fluorocytosine at $10^{-7} \mathrm{M}$ to $10^{-5} \mathrm{M}$ were of the same order as the growth rate inhibition data for these compounds (Tables 1 and 2), whereas the data for the azole antifungals suggested they often inhibited hyphal length more than growth rate. This difference was accounted for by an increased tendency for the mycelia to initiate yeast cell growth by lateral budding in the presence of azole compounds (Table 3), and by an increase in average hyphal diameter of 12 to $32 \%$. The numbers of secondary yeast cells and the increase in hyphal diameter were usually greatest at the highest antifungal concentrations tested. Only ICI 153066 and itraconazole at $10^{-5} \mathrm{M}$ caused a relatively small increase in numbers of non-primary yeast cells. This observation correlated with the data on hyphal length inhibition by these two compounds (Tables 2 and 3). 
Table 3. Effects of antifungal drugs on numbers of non-primary yeast cells per C. albicans mycelium after 7.5 hrowth in EMEM

Cultures that were not treated with drugs contained a mean of $0 \cdot 15 \pm 0.03$ yeast cells per mycelium. Standard deviations for experiments with three or more replicates ranged from $0 \cdot 00$ to $0 \cdot 07$; NT, not tested:- - growth was totally inhibited, no mycelium formed.

\begin{tabular}{|c|c|c|c|c|c|c|}
\hline Antifungal & Concn (M) & $10^{-9}$ & $10^{-8}$ & $10^{-7}$ & $10^{-6}$ & $10^{-5}$ \\
\hline Amphotericin B & & NT & NT & $0 \cdot 2$ & - . & $\ldots$ \\
\hline 5-Fluorocytosine & & NT & NT & $0 \cdot 1$ & $0 \cdot 2$ & 0.7 \\
\hline Clotrimazole & & $0 \cdot 3$ & $1 \cdot 8$ & $2 \cdot 2$ & $2 \cdot 8$ & $3 \cdot 5$ \\
\hline ICI 153066 & & $0 \cdot 2$ & 0.7 & 0.7 & $0 \cdot 6$ & $0 \cdot 6$ \\
\hline Itraconazole & & 0.2 & 0.5 & $0 \cdot 6$ & 0.6 & 1.7 \\
\hline Ketoconazole & & $0 \cdot 2$ & 1.5 & $2 \cdot 2$ & $3 \cdot 6$ & $4 \cdot 0$ \\
\hline Miconazole & & $0 \cdot 1$ & 0.8 & $2 \cdot 1$ & $4 \cdot 8$ & $4 \cdot 4$ \\
\hline Tioconazole & & 0.2 & 1.7 & 1.6 & 1.6 & $5 \cdot 3$ \\
\hline
\end{tabular}

Table 4. Effects of antifungal drugs on hyphal branching of C. albicans

The values are percentage reductions in numbers of branches per mycelium measured after $7.5 \mathrm{~h}$ growth in EMEM. Data are the means of at least duplicate experiments. Standard deviations for experiments with three or more replicates ranged from $2 \%$ to $9 \%$; NT, not tested.

\begin{tabular}{|c|c|c|c|c|c|c|}
\hline Antifungal & Conen (M) . & $10^{-9}$ & $10^{-8}$ & $10^{-7}$ & $10^{-6}$ & $10^{-5}$ \\
\hline Amphotericin B & & NT & NT & 35 & 100 & 100 \\
\hline 5-Fluorocytosine & & NT & NT & 23 & 88 & 100 \\
\hline Clotrimazole & & 0 & 92 & 97 & 99 & 99 \\
\hline ICI 153066 & & 4 & 86 & 97 & 97 & 99 \\
\hline Itraconazole & & 25 & 95 & 88 & 94 & 97 \\
\hline Ketoconazole & & 74 & 98 & 94 & 96 & 98 \\
\hline Miconazole & & 23 & 91 & 97 & 95 & 100 \\
\hline Tioconazole & & 30 & 100 & 98 & 98 & 99 \\
\hline
\end{tabular}

\section{Effects of antifungals on hyphal branching of C. albicans}

At least a $23 \%$ reduction in the number of branches formed per mycelium was observed at all azole concentrations tested except for clotrimazole and ICI 153066, which appeared not to affect branching at $10^{-9} \mathrm{M}$. For all azole compounds, hyphal branching was inhibited by 86 to $100 \%$ at concentrations from $10^{-8} \mathrm{M}$ to $10^{-5} \mathrm{M}$ (Table 4). Inhibition of branch formation of this order was seen with amphotericin $B$ and 5-fluorocytosine only at concentrations where growth rates were inhibited by $50 \%$ or more (Tables 1 and 4 ). The data in Table 4 are amplified by the detailed time courses of branch formation shown for one of the test compounds in Fig. 2(b). The time at which branching commenced was the same in the presence and in the absence of the antifungals, but the extent to which branches were formed was markedly reduced in the presence of the azole compounds.

\section{Effects of antifungals on secondary hyphal outgrowths from parent yeast cells}

Of all the inhibitory effects of azole antifungals on $C$. albicans hyphae, prevention of outgrowths of secondary and later hyphae from the original parent yeast cells was the property that revealed variation in the effects of the individual compounds (Table 5). Clotrimazole and miconazole reduced the number of hyphal outgrowths by more than $70 \%$ at concentrations of $10^{-7} \mathrm{M}$ and higher. Ketoconazole and tioconazole were somewhat less inhibitory than clotrimazole and miconazole at $10^{-7} \mathrm{M}$, but they were similarly inhibitory at $10^{-8} \mathrm{M}$. ICI 153066 and itraconazole had relatively little effect on secondary hyphal outgrowths at concentrations below $10^{-5} \mathrm{M}$. Amphotericin B and 5-fluorocytosine had this particular inhibitory effect only at concentrations that also caused substantial inhibition of biomass increase (Table 1). 
Table 5. Effects of antifungal drugs on secondary hyphal outgrowths from parent yeast cells of C. albicans

The values are percentage reductions in numbers of secondary hyphal outgrowths per mycelium. Standard deviations for experiments with three or more replicates ranged from $1 \%$ to $11 \%$ : NT, not tested.

\begin{tabular}{lrrrrr}
\multicolumn{1}{c}{ Antifungal } & Concn (M) ... $10^{-4}$ & $10^{-8}$ & $10^{-7}$ & $10^{-6}$ & $10^{-5}$ \\
Amphotericin B & NT & NT & 42 & 100 & 100 \\
5-Fluorocytosine & NT & NT & 4 & 22 & 83 \\
Clotrimazole & 3 & 75 & 75 & 80 & 100 \\
ICI 153066 & 1 & 0 & 26 & 33 & 42 \\
Itraconazole & 0 & 12 & 4 & 26 & 64 \\
Ketoconazole & 35 & 57 & 55 & 75 & 77 \\
Miconazole & 25 & 45 & 71 & 75 & 94 \\
Tioconazole & 9 & 52 & 55 & 54 & 93
\end{tabular}

Table 6. Effects on biomass growth rates (measured in terms of ATP concentration) and hyphal branch formation of time of addition or removal of $10^{-7} \mathrm{M}$-ketoconazole in cultures of $C$. albicans grown in EMEM

For details of replication data see Table $4 ;$ NT, not tested. Time of
addition or
removal of
ketoconazole

(h)

0

1

2

4

6
Ketoconazole added

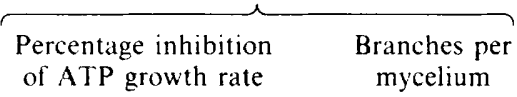

18

NT

7

7

4 mycelium

100

NT

69

31

19

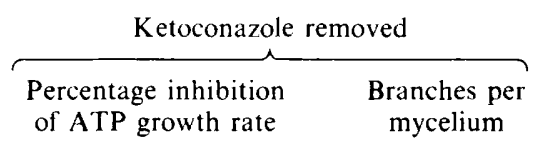

NT
14
10
14
NT

43

50

64

NT

Significance of time of additions or remoral of antifungal on development of C. albicans hyphae

Ketoconazole at $10^{--} \mathrm{M}$ was selected as representative of the effects of azole antifungals on $C$. albicans hyphal development to investigate the effects of addition or removal of the drug at various times before and after the 'zero time' additions used in the previously described experiments. Inhibition of hyphal branching and growth rate were measured as the most sensitive indicators of the range of effects of ketoconazole. The results (Table 6) suggested that ketoconazole was an effective inhibitor only when it was present in the culture. Its addition at times later than $0 \mathrm{~h}$ caused progressively less inhibition of growth rate and branch formation. When the antifungal was removed from the culture at intervals its inhibitory effects on biomass growth rate and branching were generally greater the longer the period of exposure of fungus to antifungal. It was, however, notable that hyphal branching was reduced by $43 \%$ even in cultures that had been exposed to ketoconazole for only the first hour of growth (Table 6): the time at which branches first appeared was at least $2 \mathrm{~h}$ later than this.

\section{DISCUSSION}

The results of this study confirm previous reports that imidazole antifungals can affect the morphological development of C. albicans hyphae (Borgers et al., 1979; Borgers, 1980; Davies \& Marriott, 1981). They show that triazole antifungals exert similar effects and they define more precisely than before the nature of the steps in morphogenesis that are affected by azole antifungal compounds. At very low concentrations, all the azole antifungals tested substantially inhibited hyphal branching. but none of them prevented emergence of a normal germ tube in the 
earliest stages of growth. Some of the compounds appeared to prevent additional hyphal outgrowths from parent yeast cells, thus favouring emergence of budding yeast cells. At the highest concentration tested, $10^{-5} \mathrm{M}$, most of the azoles tested caused substantial inhibition of hyphal length with concomitant enforcement of growth in the shape of budding yeast cells. The yeast cells commonly appeared not to separate, suggesting that the non-separation mechanism characteristic of hyphae might still be operating, but this possibility was not specifically investigated. Although none of these morphological effects were fully expressed in the absence of some inhibition of hyphal growth rate, the extent of inhibition of growth rate was substantially less than that of the morphological changes. The two control non-azole antifungals amphotericin B and 5-fluorocytosine had similar apparent morphological effects only when hyphal growth rate was also substantially reduced, and these effects are therefore considered non-specific to morphogenesis.

Two primary modes of action have been proposed for azole antifungals against $C$. albicans. The first is an effect on cytochrome P-450 (Van den Bossche \& Willemsens, 1982), which is probably the cause of an inhibition of ergosterol biosynthesis that results in accumulation of 14$\alpha$-methyl sterols in the plasma membrane. The second effect is a direct interaction of the drugs with membrane lipids, leading to membrane damage (Van den Bossche et al., 1983), which is probably the result of changes in the lipid transition temperature (Van den Bossche et al., 1982). Interaction with cytochrome P-450 and inhibition of ergosterol synthesis in yeast cells have been demonstrated for several azoles at concentrations of the order of $5 \times 10^{-8} \mathrm{M}$ to $5 \times 10^{-7} \mathrm{M}$ (Marriott, 1980; Van den Bossche \& Willemsens, 1982; Van den Bossche et al., 1978, 1980, 1982) whereas direct membrane damage requires higher azole concentrations - at least $10^{-6} \mathrm{M}$ and usually $10^{-5}$ M or more (Sreedhara Swamy et al., 1974; Cope, 1980; Sud \& Feingold, 1981a, 1981 b; Uno et al., 1982; Van den Bossche et al., 1983, 1984). A slow-acting inhibitory effect of miconazole and ketoconazole on membrane fatty acid synthesis in $C$. albicans has been proposed, but little of the evidence for this has been formally published (Van den Bossche et al., 1983).

The lowest antifungal concentrations known to have an effect on the cytochrome P-450 target in cell-free systems are of precisely the same order as the lowest concentrations that affected branch development of $C$. albicans in the present study (Table 4). Inhibition of growth rate (Table 1) was lowest at the lowest antifungal concentrations; some inhibition of growth rate at concentrations as low as $10^{-8} \mathrm{M}$ has been noted before (Van den Bossche et al., 1980).

The impression gained is therefore that the effects of the azole antifungals on hyphal branching may be related mainly to the effects of the drugs on cytochrome P-450. Because inhibition of hyphal extension and secondary hyphal shoot formation are seen only at higher drug concentrations and principally with drugs known to possess the capability for direct membrane damage, these effects probably result mainly from direct interactions of the compounds with the fungal cell membranes. All the known membrane changes caused directly and indirectly in azole-treated C. albicans are likely to alter the fluidity of the plasma membrane (Van den Bossche et al., 1983). Because several of the enzymes involved in C. albicans cell wall synthesis are located in the cell membrane (Braun \& Calderone, 1978; Marriott, 1975; Chiew et al., 1980) it is possible that changes in membrane fluidity could affect the activity of these enzymes and their regulation, and thus lead to distorted cell wall synthesis with concomitant changes in cell shape. The fact that the azole compounds at low concentrations have an effect selectively related to hyphal branching presumably depends on a different mechanism, possibly related to the known effects of azole antifungals on cytochrome P-450 and sterol synthesis.

In some fungi with an entirely hyphal mode of development, such as Neurospora crassa, regulation of branch formation has been shown to depend on a $\mathrm{Ca}^{2+} /$ cyclic AMP mechanism, which is affected by ionophores such as A23187 (Reissig \& Kinney, 1983). The possibility that azole antifungals affect a similar mechanism awaits investigation. It has been postulated that hyphal branching of $C$. albicans is a process regulated by gene expression whereas overall cell shape is regulated mainly at the phenotypic level by environmental influences on cell wall synthesizing enzymes (Odds, 1985). The fact that the azole compounds tested in the present 
study were shown to affect these two aspects of cell development differentially supports the notion that they are not regulated by a common process. Further experiments are needed to establish which cell wall synthesizing enzymes and intracellular proteins are affected by azole antifungals at different stages of mycelium formation in $C$. albicans.

We gratefully acknowledge the support of a Medical Research Council studentship for one of us (A.C.).

\section{REFERENCES}

Ansehn, S. \& Nilsson, L. (1984). Direct membranedamaging effect of ketoconazole and tioconazole on Candida albicans demonstrated by bioluminescent assay of ATP. Antimicrobial Agents and Chemotherapy 26, 22-25.

BoRGERS, M. (1980). Mechanism of action of antifungal drugs, with special reference to the imidazole derivatives. Review's of Infectious Disease 2, 520534.

Borgers, M., Van den Bossche, H., De Brabander, M. \& VAN CUTSEM, J. (1979). Promotion of pseudomycelium formation of Candida albicans in culture: a morphological study of the effects of miconazole and ketoconazole. Postgraduate Medical Journal 55, 687-69l.

Braun, P. C. \& Calderone, R. A. (1978). Chitin synthesis in Candida albicans: comparison of yeast and hyphal forms. Journal of Bacteriology 133, 1472 1477.

Chiew, Y. Y., Shepherd, M. G. \& Sullivan, P. A (1980). Regulation of chitin synthesis during germtube formation in Candida albicans. Archives of Microbiology 125, 97-104.

COPE, J. E. (1980). Mode of action of miconazole on Candida albicans: effect on growth, viability and $\mathrm{K}^{+}$ release. Journal of General Microbiology 119, 245251.

Davies, A. R. \& Marriott, M. S. (1981). Inhibitory effects of imidazole antifungals on the yeastmycelial transformation in Candida albicans. Microbios Letters 17, 155-158.

JohnSON, E. M., Barnard, M. L., RICHARDSON, M. D. \& WARNOCK, D. W. (1982). Effect of ketoconazole on the initial stages of germ tube formation by strains of Candida albicans. Mykosen 25, 481-486.

MARRIOTT, M. S. (1975). Enzymic activity of purified plasma membranes from the yeast and mycelial forms of Candida albicans. Journal of General Microbiology 89, 345-352.

MARRIOTT, M.S. (1980). Inhibition of sterol biosynthesis in Candida albicans by imidazole-containing antifungals. Journal of General Microbiology 117, 253255.

ODDS, F. C. (1982). Interactions among amphotericin B, 5-fluorocytosine, ketoconazole and miconazole against pathogenic fungi in vitro. Antimicrobial Agents and Chemotherapy 22, 763-770.

ODDs, F. C. (1985). Morphogenesis in Candida albicans. CRC Critical Review's in Microbiology 12, 45-93.
Reissig, J. L. \& Linney, S. G. (1983). Calcium as a branching signal in Neurospora crassa. Journal of Bacteriology 154, 1397-1402.

Sreedhara Swamy, K. H., Sirsi, M. \& Rao, G. R (1974). Studies on the mechanism of action of miconazole: effect of miconazole on respiration and cell permeability of Candida albicans. Antimicrobial Agents and Chemotherapy 5, 420-425.

Sud, I. J. \& FeINGOLD, D. S. (1981 $a$ ). Heterogeneity of action mechanisms among antimycotic imidazoles. Antimicrobial Agents and Chemotherapy 20, 71-74.

Sud, I. J. \& FEINGOLD, D. S. (1981 $b$ ). Mechanisms of action of the antimycotic imidazoles. Journal of Investigative Dermatology 76, 438-441.

Uno, J., Shigematsu, M. L. \& ARaI, T. (1982). Primary site of action of ketoconazole on Candida albicans. Antimicrobial Agents and Chemotherapy 21, 912-918.

VAN deN Bossche, H. \& Willemsens, G. (1982). Effects of the antimycotics, miconazole and ketoconazole, on cytochrome $\mathrm{P}-450$ in yeast microsomes and rat liver microsomes. Archices Internationales de Physiologie et Biochemie 90, B218-B219.

Van den Bossche, H., Willemsens, G., Cools, W., Lauwers, W. F. J. \& LeJeune, L. (1978). Biochemical effects of miconazole on fungi. II. Inhibition of ergosterol biosynthesis in Candida albicans. In Chemical-Biological Interactions, vol. 21, pp. 59-78. Amsterdam: Elsevier.

Van den Bossche, H., Willemsens, G., Cools, W. Cornelissen, F., Lauwers, W. F. \& Van Cutsem, J. M. (1980). In vitro and in vivo effects of the antimycotic drug ketoconazole on sterol synthesis. Antimicrobial Agents and Chemotherapy 17, 922-928.

VAN DEN Bossche, H., RuYsSChaerT, J. M., DefriseQuertain, F., Willemsens, G., Cornelissen, F., Marichal, P., Cools, W. \& Van Cutsem, J. (1982). The interaction of miconazole and ketoconazole with lipids. Biochemical Pharmacology 31, 26092617.

Van den Bossche, H., Willemsens, G., Cools, W., MARICHAL, P. \& LAUWERS, W. (1983). Hypothesis on the molecular basis of the antifungal activity of $\mathrm{N}$-substituted imidazoles and triazoles. Biochemical Society Transactions 11, 665-667.

VAN DEN BossChe, H., LAUWERS, W., Willemsens, G., Marichal, P., Cornelissen, F. \& Cools, W. (1984). Molecular basis for the antimycotic and antibacterial activity of $\mathrm{N}$-substituted imidazoles and triazoles: the inhibition of isoprenoid biosynthesis. Pesticide Science 15, 188-198. 